\title{
Distinguishing potential bacteria-tumor associations from contamination in a secondary data analysis of public cancer genome sequence data
}

Kelly M. Robinson ${ }^{1}$, Jonathan Crabtree', John S. A. Mattick', Kathleen E. Anderson ${ }^{1}$ and Julie C. Dunning Hotopp ${ }^{1,2,3^{*}}$

\begin{abstract}
Background: A variety of bacteria are known to influence carcinogenesis. Therefore, we sought to investigate if publicly available whole genome and whole transcriptome sequencing data generated by large public cancer genome efforts, like The Cancer Genome Atlas (TCGA), could be used to identify bacteria associated with cancer. The Burrows-Wheeler aligner (BWA) was used to align a subset of Illumina paired-end sequencing data from TCGA to the human reference genome and all complete bacterial genomes in the RefSeq database in an effort to identify bacterial read pairs from the microbiome.

Results: Through careful consideration of all of the bacterial taxa present in the cancer types investigated, their relative abundance, and batch effects, we were able to identify some read pairs from certain taxa as likely resulting from contamination. In particular, the presence of Mycobacterium tuberculosis complex in the ovarian serous cystadenocarcinoma (OV) and glioblastoma multiforme (GBM) samples was correlated with the sequencing center of the samples. Additionally, there was a correlation between the presence of Ralstonia spp. and two specific plates of acute myeloid leukemia (AML) samples. At the end, associations remained between Pseudomonas-like and Acinetobacter-like read pairs in AML, and Pseudomonas-like read pairs in stomach adenocarcinoma (STAD) that could not be explained through batch effects or systematic contamination as seen in other samples.

Conclusions: This approach suggests that it is possible to identify bacteria that may be present in human tumor samples from public genome sequencing data that can be examined further experimentally. More weight should be given to this approach in the future when bacterial associations with diseases are suspected.
\end{abstract}

Keywords: Microbiome, Cancer, Batch effects, Genome sequencing, Cancer-associated bacteria, Acinetobacter, Pseudomonas, Stomach adenocarcinoma, Acute myeloid leukemia

\footnotetext{
* Correspondence: jdhotopp@som.umaryland.edu

${ }^{1}$ Institute for Genome Sciences, University of Maryland School of Medicine,

Baltimore, MD, USA

${ }^{2}$ Department of Microbiology and Immunology, University of Maryland

School of Medicine, Baltimore, MD, USA

Full list of author information is available at the end of the article
} 


\section{Background}

Given that bacteria live in and on our bodies, they have a large potential to affect human health. An estimated $15-20 \%$ of cancers worldwide are linked to viral, parasitic, or bacterial infections that were responsible for 1.5 million cancer deaths in 2008 [1]. The best-studied examples of cancer-associated infectious agents are hepatitis B virus (HBV), human papillomavirus (HPV), Epstein-Barr virus (EBV), human immunodeficiency virus (HIV), Schistosoma haematobium, and Helicobacter pylori. A subset of these viruses are known to integrate into the human genome [2, 3], while viruses, parasites, and bacteria can all promote cancer through other mechanisms [4].

Of the bacteria known to be associated with carcinogenesis, the mechanisms linking $H$. pylori to gastric carcinoma and gastric mucosa-associated lymphoid tissue (MALT) lymphoma are best understood [5]. In addition to $H$. pylori, many other microbes have been associated with various carcinomas [6]. For example, S. haematobium with bladder carcinoma [7], Salmonella typhi with gallbladder cancer [8], Chlamydia pneumoniae with lung cancer [9], Bacteroides fragilis [10, 11] and Streptococcus bovis [12] with colon cancer, and Escherichia coli [13] and Fusobacterium spp. [14-18] with colorectal cancer. Frequently, bacteria are thought to contribute to carcinogenesis through increased inflammation, which promotes DNA damage [19]. While most cancer-related bacteria are the dominant member of the microbiome, it is possible that rare members could cause driver mutations and/or that dominant members might be more abundant in tumors due to a favorable tumor microenvironment.

S. haematobium is a parasitic flatworm classified as a definite carcinogen $[7,20]$ and was one of the first associations identified between an infectious agent and cancer formation [21]. The flatworm lays its eggs in the bladder mucosa causing constant irritation and inflammation [22] that is exacerbated when some eggs cannot be excreted through the urine and become trapped in the tissue [23]. The ability of S. haematobium to increase inflammation [22-24], decrease apoptosis [20, 23], and increase cell proliferation $[20,23]$ are the reasons for its classification as a definite carcinogen.

$H$. pylori also increases host inflammation and was the first bacterial species to be considered a carcinogen by the International Agency for Research on Cancer [25]. H. pylori is present in $90 \%$ of non-cardia gastric cancer cases and $86 \%$ of gastric MALT lymphoma cases [1]. $H$. pylori can alter host signaling pathways [25] and methylation of host genes [26]. Infections by $H$. pylori strains containing the cytotoxin-associated gene (cag) pathogenicity island cause upregulation of the mitogenic signaling pathway through activation of the $c$-fos and c-jun proto- oncogenes [25, 27], in addition to altering a number of other pathways.

The bioinformatics pipeline PathSeq [28] was used to identify an increase in Fusobacterium sequences in data from DNA [14] and rRNA-depleted RNA [15] from colorectal cancer samples relative to normal samples, as well as a decrease of Bacteroidetes and Firmicutes phyla in these colorectal cancer samples [14]. These findings were subsequently confirmed with $16 \mathrm{~S}$ rRNA gene analysis and quantitative PCR on a larger group of samples, as well as using FISH to visualize the bacteria within the tumor cells [14]. While not definitively demonstrating that a Fusobacterium sp. can cause cancer, these results prompted more consideration for using sequencing data to identify candidate bacteria involved in carcinogenesis, without relying on culture-based techniques.

As more genome sequencing data becomes available, secondary, retrospective studies can be carried out to test other hypotheses. However, such studies are not without biases since the analysis is conducted looking through a lens that can often be clouded with uncertainty related to sequencing type, unknown metadata factors, and lack of access to original samples. For example, bias can be introduced from the type of sequencing undertaken, which is not always clear in associated metadata or text on methods in publications. For example, human RNA sequencing (RNA-Seq) data is often from a library constructed from poly-A-selected RNA, which removes the RNA of many but not all bacteria. Therefore, it is impossible to determine which bacteria may be present in the sample, but missing from poly-A-selected data. For example, The Cancer Genome Atlas (TCGA) analysis of the poly-A-selected RNA-Seq stomach adenocarcinoma (STAD) data detected $H$. pylori only sporadically, which they attribute to either the decline of bacterial abundance upon progression from chronic gastritis to subsequent carcinoma or the technical loss of luminal bacteria during specimen processing [29].

Using a method analogous to PathSeq, we previously presented evidence supporting the presence of various microbes in tumor samples from TCGA. This study predominantly focused on identifying bacteria-human lateral gene transfer (LGT) events in a subset of TCGA data (Table 1). Putative bacteria-human LGT events were found in tumor suppressor and proto-oncogenes in stomach adenocarcinoma samples, as well as in the mitochondrial genome of acute myeloid leukemia samples (AML) [30]. In these cases, it was reported that the microbiome-associated bacteria of the samples with putative LGTs were highly reflective of the bacterial species from which the putative LGT originated [30]. Specifically, the STAD samples with putative Pseudomonas-like integrations had Pseudomonas spp. as the 
Table 1 List of cancer types analyzed, their TCGA designated abbreviations, and the sequencing type analyzed

\begin{tabular}{lll}
\hline Cancer type & Abbreviation & Sequencing type \\
\hline Acute myeloid leukemia & AML & RNA-Seq (192) \\
Breast cancer & BRCA & RNA-Seq (451) \\
Glioblastoma multiforme & GBM & RNA-Seq (1), DNA (10), \\
Kidney clear cell carcinoma & KIRC & RNA-Seq (420) \\
Kidney papillary carcinoma & KIRP & RNA-Seq (15) \\
Lung adenocarcinoma & LUAD & RNA-Seq (76) \\
$\begin{array}{l}\text { Lung squamous cell carcinoma } \\
\text { Ovarian serous } \\
\text { cystadenocarcinoma }\end{array}$ & LUSC & RNA-Seq (174) \\
Stomach adenocarcinoma & OV & WGA (310), DNA (13) \\
\hline
\end{tabular}

${ }^{1}$ As denoted by the TCGA barcode, the sequencing types are RNA-Seq, DNA, or whole genome amplified (WGA). The number of samples analyzed for each sequencing type is denoted in the parentheses and is not the number of samples where bacterial read pairs were identified

predominant member of their microbiome, while the STAD samples lacking LGT had a higher relative abundance of Enterobacteriaceae [30]. Also, the AML samples with putative Acinetobacter-like DNA integrations had an increased relative abundance of microbiomeassociated Acinetobacter sequences [30].

We sought to more systematically examine the bacterial sequences detected across numerous sample types in TCGA data and to develop techniques to distinguish the presence of biologically/medically significant microbes from the presence of bacterial contamination, which can arise in numerous ways. For example, the presence of bacterial DNA in laboratory kits and reagents was found to impact the results of $16 \mathrm{~S}$ rRNA gene and shotgun metagenomics methods, particularly samples with a low microbial biomass [31]. Microbial contamination has been identified in nucleic acid extraction spin columns, where column contamination during manufacturing was responsible for the discovery of a novel DNA virus [32]. Another example of microbial contamination in a human genomics project was the identification of HPV-18 in cervical cancer and nine other cancer types from TCGA [33]. This was determined to be contamination in the non-cervical cancer samples when it was realized that the HPV-18 reads had characteristics of the typical HPV-18 integration in HeLa cells and lacked reads aligning to the regions of the HPV-18 genome that are typically not integrated [33]. Therefore, the presence of HPV-18 in the non-cervical cancer TCGA data suggests that HeLa cells which contain this HPV-18 integration were actually the cause of the contamination [33]. Additionally, contamination from liver cancer samples was the source of $\mathrm{HBV}$ in one kidney cancer TCGA sample [34].
Here, we present the results of our analysis of an early release of TCGA data for evidence of bacteria. This examination reveals that some bacterial sequences can be attributed to systematic contamination of all samples or to batch effects likely associated with contamination, while other bacteria are associated with particular tumor types without an obvious source of contamination. Since we cannot completely rule out contamination as a source of these sequences, these bacteria-tumor associations should bear closer scrutiny in future studies aimed at examining the roles bacteria might play in oncogenesis.

\section{Results}

\section{Bacterial presence in TCGA data}

In order to determine the microbial component of various cancers, we analyzed the relative abundance (subsequently referred to as abundance) of bacteria-derived paired-end Illumina sequencing in TCGA data that was made available in the Sequence Read Archive (SRA), as previously described [30]. Briefly, reads were aligned to the human genome and RefSeq with Burrows-Wheeler aligner (BWA) ALN/SAMPE, a likely taxonomic assignment was made for each read pair (Additional file 1: Figure S1), and the results (Additional file 2) were loaded into a database for analysis and visualization with Krona [35] and MicroView, which can be found at http://microview.igs.umaryland.edu/tcga_v1/. MicroView is an interactive website and derivative of our LGTview [30], which enables the interrogation of metadata using a graphical interface connected to an underlying database of raw data for each bacterial read pair (Additional file 2). MicroView is referenced in the remainder of the manuscript to denote when data was mined from MicroView that could not be represented in an individual figure. An operational taxonomic unit (OTU) is assigned to each read and read pair that should be considered approximate, as the assignment is likely to be influenced by the genomes in RefSeq. Therefore, for example, Pseudomonas reads should be considered Pseudomonaslike reads.

Nine cancer types were examined (Table 1) and found to have differing abundances of putative bacterial read pairs ranging from $11 \%$ of patients with at least one sample containing bacterial read pairs in lung squamous cell carcinoma (LUSC) to $100 \%$ of patients having at least one sample with bacterial read pairs in STAD (Table 2). STAD, AML, and ovarian serous cystadenocarcinoma (OV) have large proportions of Pseudomonas, Acinetobacter, and Mycobacterium read pairs, respectively (Fig. 1), which were not frequently identified in other cancer types (MicroView). Despite different locations in the human body, read pairs from particular bacterial taxa were repeatedly present in most cancer types 


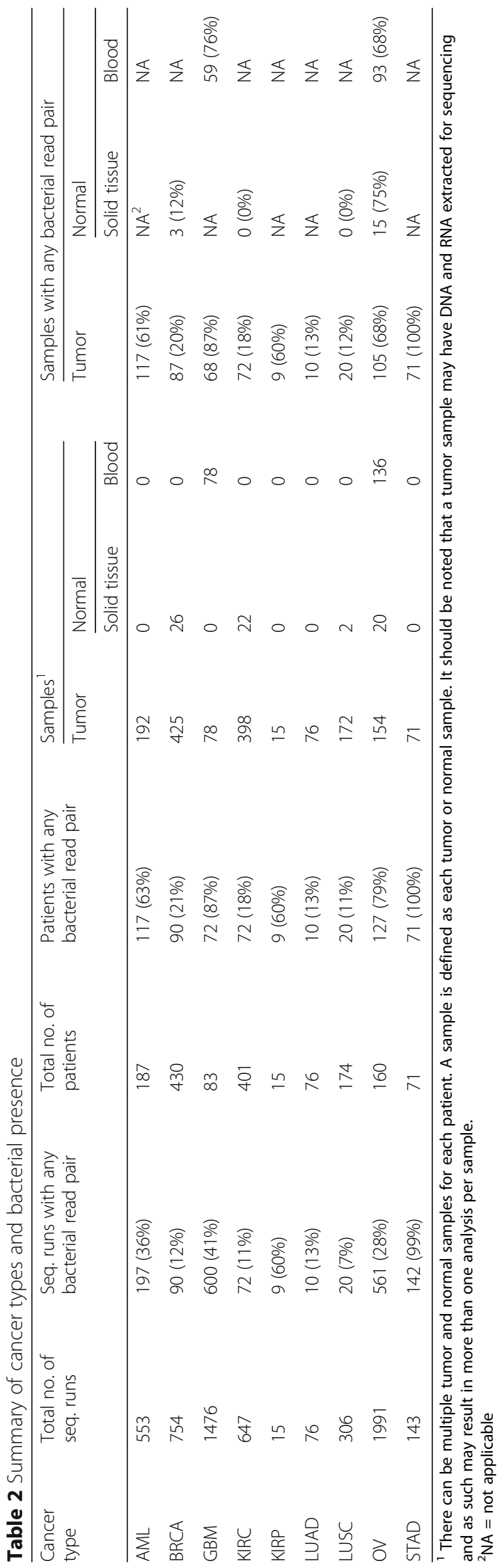




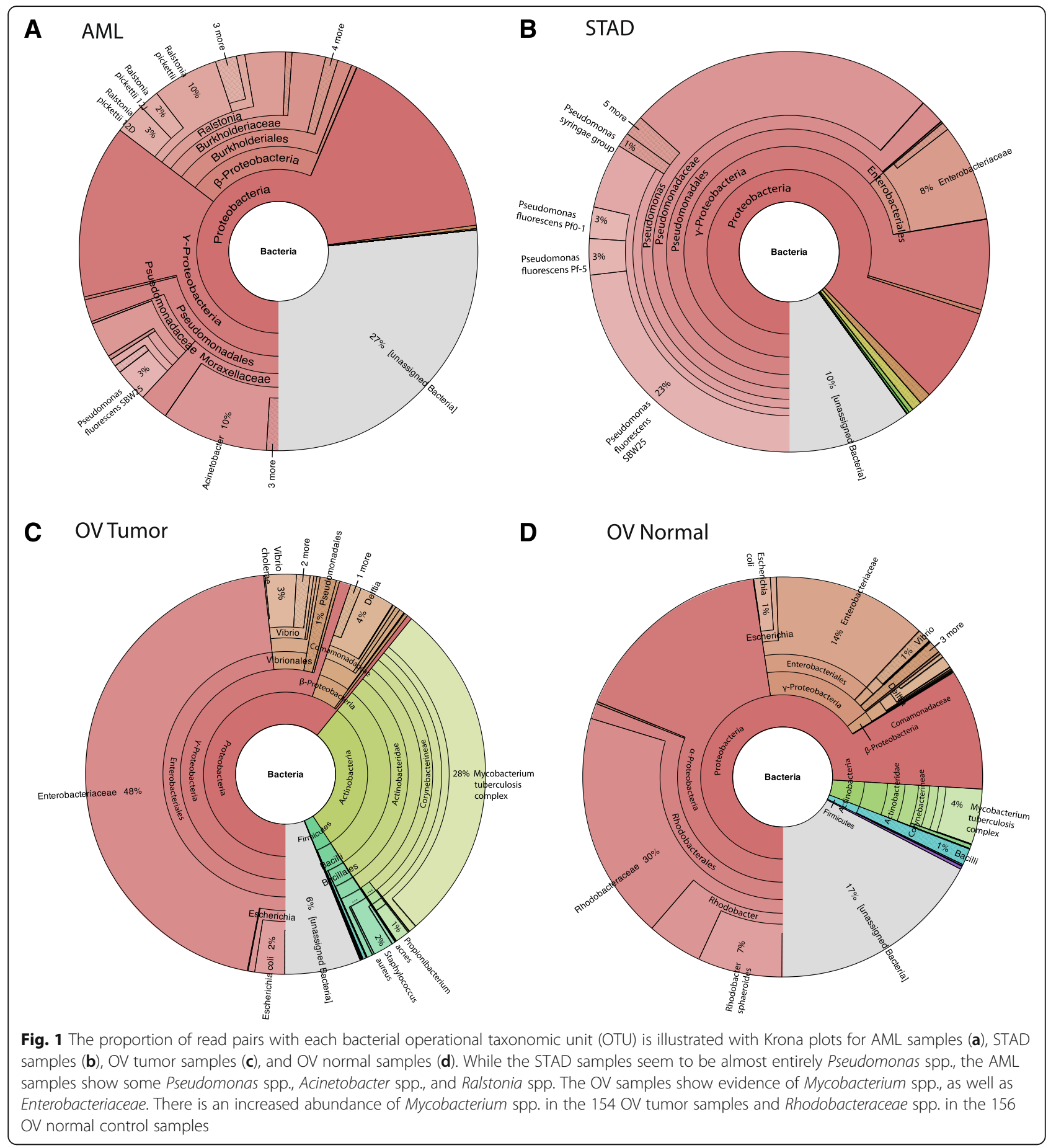

(e.g., Enterobacteriaceae, Propionibacterium, Ralstonia, and Staphylococcus taxa) (MicroView).

\section{Dominant bacteria in TCGA sequencing projects}

All of the cancer types investigated had one or more samples containing read pairs present that were assigned to bacterial taxa (Table 2). However, only STAD and kidney papillary carcinoma (KIRP) had more sequencing runs with bacterial read pairs than sequencing runs without any bacterial read pairs present (Table 2, Additional file 3: Figure S2). Some bacterial species were the dominant taxa of a specific cancer type, as was the case with Pseudomonas in STAD, Acinetobacter in AML, and Mycobacterium tuberculosis complex in OV tumor samples (Fig. 1). In the STAD samples, $62 \%$ of the bacterial read pairs were assigned as 
Pseudomonas (Fig. 1), although most of the read pairs are found in only a few samples (Fig. 2). In contrast, the AML samples were dominated by $7 \%$ Pseudomonas, 10\% Acinetobacter, and 15\% Ralstonia read pairs (Fig. 1). Although kidney clear cell carcinoma (KIRC) also had Pseudomonas read pairs present in the samples, it was responsible for $10 \%$ of the total bacterial read pairs (MicroView). The OV samples surprisingly revealed evidence of Mycobacterium read pairs, as well as Enterobacteriaceae read pairs (Fig. 1). The main difference in bacterial proportions when comparing the tumor and healthy tissue samples for $\mathrm{OV}$ is the increased abundance of Mycobacterium read pairs in the tumor samples, as well as the presence of Rhodobacteraceae read pairs in the normal samples (Fig. 1). A similar comparison for AML and STAD cannot be made, as no normal samples were available.

In general, samples of DNA and whole-genome amplified DNA were clustered together regardless of sequencing center or cancer type since samples were sequenced by both The Broad Institute of MIT and Harvard (Broad) and Washington University in St. Louis from OV and glioblastoma multiforme (GBM) (Fig. 2). DNA samples had a lower mapped read count relative to the RNA samples. However, while the DNA samples are in one distinct cluster, the RNA samples formed many distinct clusters that at times relate to cancer type (e.g., AML) (Fig. 2). However, AML samples had multiple distinct groups (Fig. 2).

\section{A comparison of aggregate bacteria found in tumor and normal samples}

Only five sets of samples in the data set we used had tumor-normal pairs. Breast cancer (BRCA), OV, and GBM had both tumor and normal samples that contained bacterial sequences. In contrast, bacterial read pairs were only present in the tumor samples for KIRC and LUSC, although LUSC only had two normal samples sequenced in this release (Table 2). While the majority of the bacterial taxa in the BRCA tumor and non-paired normal samples were similar, there were some minor

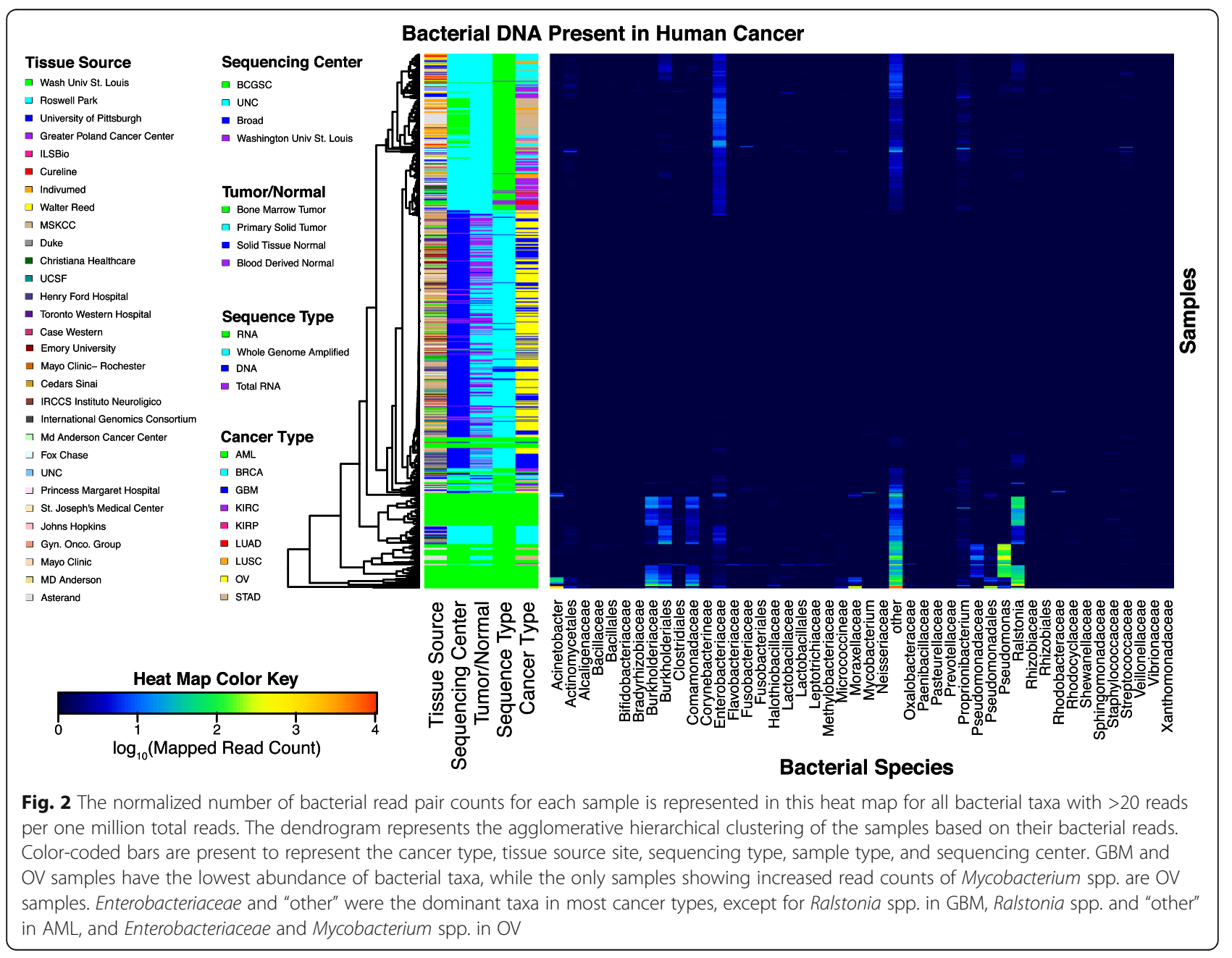


differences (MicroView). However, it is important to note that only 3 of 26 normal samples and 87 of 425 tumor samples contained bacterial read pairs at the time this data was downloaded and analyzed (Table 2). From the samples containing bacteria, the normal samples only had 4,526 bacterial read pairs compared to 983,611 bacterial read pairs from the tumor samples (Table 2). The GBM dataset had approximately the same number of bacterial read pairs per sample for both the tumor and paired normal samples (Table 2). Both the tumor and normal samples had about a third of the read pairs from Ralstonia picketti with low levels of read pairs from Enterobacteriaceae, Pseudomonas aeruginosa, and $M$. tuberculosis complex (Additional file 4: Figure S3). There was a shift in the proportion of Propionibacterium acnes read pairs with an $\sim 10 \%$ increase in the aggregate for the normal GBM samples (Additional file 4: Figure S3). Additionally, there is a difference in the percentage of read pairs classified as "unassigned bacteria" between the GBM tumor and normal samples (Additional file 4: Figure S3). This may happen, in general, if the bacterial read pairs in one sample align to parts of conserved regions of bacterial genomes, thus making it more difficult to determine a specific taxon to assign to the read pair (Additional file 1: Figure S1). Overall, these tumor-normal pairs clustered together in the heat map, indicating their similarity (Fig. 2). Due to the similarity of the tumor and normal samples, in the remainder of this analysis, they will be grouped together by cancer type, except when explicitly noted.

\section{Mycobacterium spp. in samples}

Upon evaluating the samples with read pairs that had assigned bacterial taxa, it was surprising that read pairs designated as $M$. tuberculosis complex could be found in both the tumor and paired-normal samples of OV and GBM (Fig. 1). In particular, bacteria are not expected in the GBM samples as this is a type of brain tumor and few bacteria can cross the blood-brain barrier. The taxonomic assignment of the Mycobacterium read pairs from the $\mathrm{OV}$ and GBM dataset was confirmed using a BLASTN search against NT, which is a more comprehensive database of reference sequences than what was used in the initial OTU assignment.

Five sequencing centers generated the data for all of the cancer types in our analysis. All of the sequencing centers with bacterial read pairs contained at least one sample with Mycobacterium spp. read pairs (MicroView). To compare results, we normalized by the total number of read pairs sequenced, using the $\log _{10}$-transformed ratio of Mycobacterium read pairs to total read pairs. A disproportionate number of OV samples sequenced at The Broad Institute had a $\log _{10}$ ratio of Mycobacterium read pairs to a total read pairs of $\geq-7$ (Table 3), meaning there was at least one Mycobacterium read pair for every ten million read pairs sequenced. The difference between samples sequenced at The Broad Institute and Washington University was considered significant by Fisher's exact test $\left(p\right.$ value $\left.=4.03 \times 10^{-7}\right)$. There was only one sample sequenced at Washington University in St. Louis that had such a large proportion of Mycobacterium read pairs, and none of the OV samples sequenced at Washington University in St. Louis contained these elevated levels of Mycobacterium spp. read pairs (Table 3 ).

The Broad Institute had a National Institute of Allergy and Infectious Diseases-funded white paper to sequence Mycobacterium spp. at approximately the same time that these TCGA samples would have been sequenced. Combined with the batch effects associated with the sequencing center, we conclude that the Mycobacterium reads most likely arose from contamination. This conclusion is similar to another contamination analysis of TCGA OV samples from The Broad Institute when dengue virus 2 was determined to be present in the sequence data due to cross-contamination from the sequencers [33]. A center that sequences many microbial genomes along with human genomes (like The Broad Institute) will have more contamination from those microbial genomes. A sequencing center that primarily sequences human samples would not have as much likelihood of experiencing these same microbial contamination issues but may have similar levels of contamination from other sources. However, overall, samples from The Broad Institute have the lowest levels of bacterial sequences when normalized for the amount of data sequenced (Fig. 2).

\section{Presence of Enterobacteriaceae in all cancer types}

Enterobacteriaceae reads, which were present in most cancer types (Fig. 2), may be a result of E. coli DNA contamination in recombinant enzymes used in wholegenome amplification and library construction. Consistent with this, we previously identified junctions of $E$. coli and human DNA in the OV data examined here that we attribute to occurring during whole genome amplification of these samples [30]. Given these concerns and the presence of $E$. coli in all cancer types, we conclude that these sequences have a high chance of arising via contamination, and as such, we did not examine them further. However, it is important to note that $E$. coli has been associated with various forms of cancer [36], and we could be overlooking an important biological observation.

\section{Potential bacterial contaminants found in samples aggregated across cancer type}

Read pairs from Staphylococcus epidermidis, P. acnes, and Ralstonia spp. were present collectively at low levels in all of the cancer types (Fig. 1, Additional file 4: Figure 


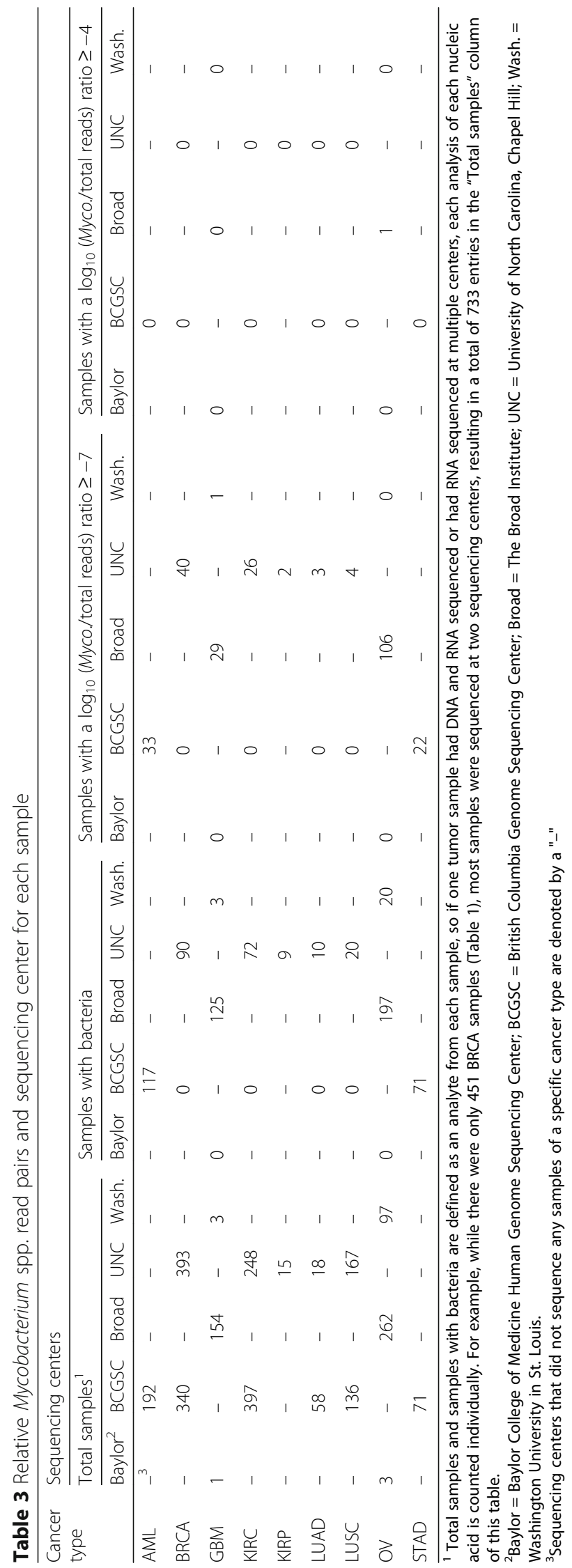


Table 4 Number of Propionibacterium spp., Ralstonia spp., and Staphylococcus epidermidis read pairs in all cancer types

\begin{tabular}{llllll}
\hline Cancer type & Total read pairs & Total bacterial read pairs & Propionibacterium read pairs & Ralstonia read pairs & $\begin{array}{l}\text { Staphylococcus epidermis } \\
\text { read pairs }\end{array}$ \\
\hline AML & $7,955,502,437$ & $29,458,420$ & 46,372 & $4,502,338$ & 9013 \\
BRCA & $6,093,925,360$ & 988,137 & 14,526 & 78,895 & 1220 \\
GBM & $5,391,069,119$ & 20,802 & 1976 & 8244 & 105 \\
KIRC & $5,070,366,679$ & 356,674 & 31,553 & 5104 & 1428 \\
KIRP & $541,775,677$ & 52,313 & 1394 & 669 & 128 \\
LUAD & $980,790,987$ & 29,583 & 534 & 914 & 28 \\
LUSC & $1,166,304,426$ & 107,515 & 12,403 & 5345 & 5714 \\
OV & $8,643,898,191$ & 34,724 & 196 & 64 & 23 \\
STAD & $6,689,562,270$ & $4,847,299$ & 31,006 & 1983 & 1323
\end{tabular}

S3, Table 4, Additional file 2), suggesting they may be contaminants. S. epidermidis and Propionibacterium spp. are skin-associated bacteria and may contaminate reagents after being shed from the skin of a laboratory and manufacturing personnel. Ralstonia spp. is thought to be a common environmental contaminant in laboratories [31].

We sought to develop a metric to identify taxa associated with a common contaminant source. Using ratios, we compared the counts of bacterial taxa to that of commonly identified bacterial taxa in these data sets, including Pseudomonas, Acinetobacter, and M. tuberculosis complex (Fig. 3). Log ratios that cluster tightly near the mean, with a low standard deviation, are taken to represent taxa clustered from a common source of contamination across multiple tumor samples. For example, the log-ratio comparing Staphylococcus spp. and Propionibacterium spp. is consistently around one for every cancer type analyzed, which means there are ten times as many Propionibacterium reads as Staphylococcus reads. As Staphylococcus spp. and Propionibacterium spp. could both be present on the human skin, this poses one mechanism for co-contamination from these two bacteria. Outliers in this ratio-based analysis suggest a different phenomenon for the two samples, which might be an alternate form of contamination or might be biologically relevant. For example, and as discussed previously, Mycobacterium reads were over-represented when compared to Staphylococcus and Propionibacterium reads in OV, with moderate ratios in GBM (Fig. 3); this likely reflects Mycobacterium contamination at the sequence center level. Other outliers include Acinetobacter in AML, Pseudomonas in STAD, and Ralstonia in AML. While Acinetobacter reads are over-represented when compared to Staphylococcus reads and Propionibacterium reads, Acinetobacter reads do not appear to be over-represented compared to Ralstonia or Pseudomonas reads in AML (Fig. 3). This suggests that there may be a relationship between Acinetobacter, Ralstonia, and Pseudomonas taxa in AML.

When a relationship is expected between taxa, like Staphylococcus and Propionibacterium, a low standard deviation is observed, indicating that they may share a common mechanism of contamination. However, other sources of contamination can alter the standard deviation, obscuring this observation. To further examine the variation of these ratios across all nine cancer types to identify a possible common mechanism, the standard deviation was calculated for these ratios based on all nine cancers and compared to the standard deviation when outlier datasets were removed, namely AML, OV, and STAD. We initially identified these three cancer types as outliers in the ratio analysis, and more specifically, it was expected that the specific higher abundances of Acinetobacter reads, Mycobacterium reads, Ralstonia reads, and Pseudomonas reads caused higher standard deviations in those comparisons. The standard deviations do decrease upon the removal of AML, OV, and STAD (Fig. 3), and we interpret this to mean that low levels of Acinetobacter reads, Ralstonia reads, and Pseudomonas reads have a common source of contamination across all samples, while high levels may arise due to an alternate explanation, which could be an alternate source of contamination. Therefore, we sought to identify further sources of contamination for Pseudomonas, Ralstonia, and Acinetobacter reads.

\section{Examination of potential bacterial contaminants found in individual samples}

In AML, as with all the cancer types, there is very little variation for the comparison of Staphylococcus reads to Propionibacterium reads (Fig. 4), which is expected for co-contaminants. In contrast, ratios including Ralstonia reads or Pseudomonas reads have a bi-modal distribution. Samples on plates 735 and 736 have a higher abundance of Ralstonia spp. (Fig. 4), suggesting that these 


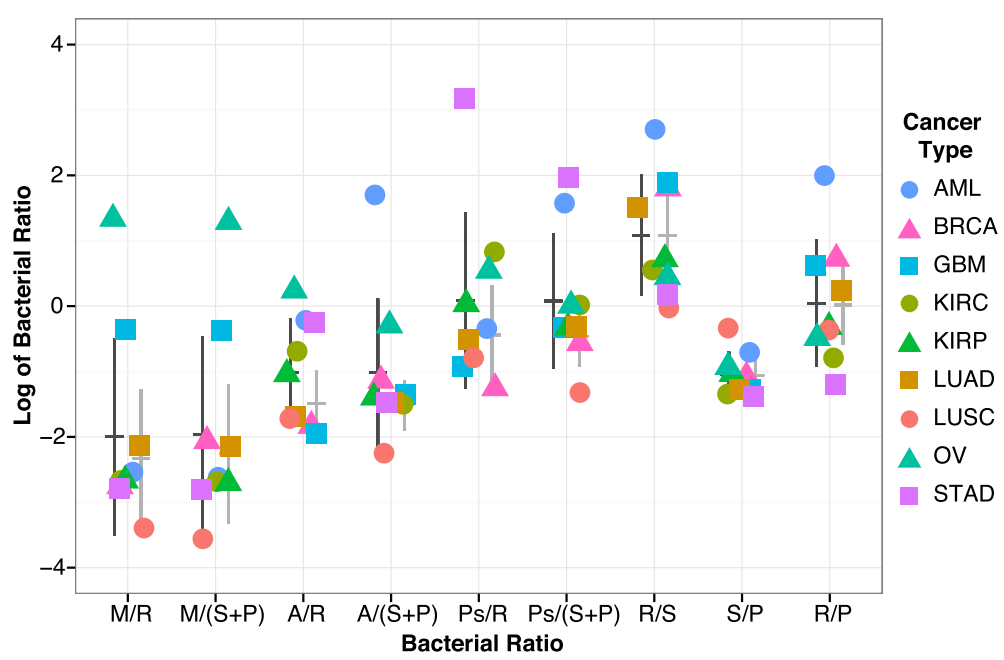

Fig. 3 The $\log _{10}$-transformed ratios of bacterial read pairs are shown for all nine cancer types comparing counts of Mycobacterium (M), Acinetobacter (A), Pseudomonas (Ps), Staphylococcus epidermidis (S), Ralstonia (R), and Propionibacterium (P) read pairs. Staphylococcus and Propionibacterium were grouped together after an initial comparison found them to frequently have the same relative proportions. In addition, they are both commonly found on the human skin and may arrive in the samples through the same mechanism. The black horizontal lines represent the average of the $\log _{10}$-transformed ratios across all the cancer types. When points are clustered near the mean from all cancer types, it suggests a common source of the bacterial reads. Given the diverse cancer types and the numerous collection and sequencing centers, we interpret those contributions to be from a general source of contamination, whereas when a set of samples does not cluster with the others (e.g., OV and GBM in M/R), it suggests a more specific source of the bacterial sequences. The latter can be due either to contamination or a biological reason, which cannot be distinguished here. This was found for comparisons containing (a) Mycobacterium in OV and GBM, (b) Pseudomonas in STAD, and (c) Acinetobacter, Ralstonia, and Pseudomonas in AML. The standard deviations with all data, depicted by the vertical black line, of each of the bacterial comparisons are shown across all nine datasets. AML, OV, and STAD, which are cancer types that have at least one predominant bacterial species, not including Enterobacteriaceae, were excluded from a subsequent mean and standard deviation calculation, depicted by the gray horizontal and vertical lines, respectively. Excluding AML, OV, and STAD decreases the standard deviation for the comparisons involving Acinetobacter spp. and Pseudomonas spp. This suggests that the presence of Acinetobacter spp. and Pseudomonas spp. may be attributed to similar levels of general contamination of all samples by a similar mechanism, except in AML, OV, and STAD

plates may have been contaminated. Upon further examination of Ralstonia reads in other cancer types, there may also be a batch effect by plate in BRCA. For example, plates A056 and A084 appear distinct from plates A00Z, A034, A109, A10J, A115, and A12D (Additional file 5: Figure S4). Despite the relationship of Ralstonia, Acinetobacter, and Pseudomonas in the AML samples described above, there is no correlation between plate and either Acinetobacter or Pseudomonas. Therefore, we conclude that Ralstonia read pairs arose from a contamination that occurred at the plate level in AML while Acinetobacter and Pseudomonas read pairs result from either a separate contamination that could not be identified or from a biologically relevant source, like the microbiome. The initial correlation may have resulted from using a single average on the aggregate study containing such broad bi-modal distributions of individual samples.

In the OV data set containing the Mycobacterium read pairs described above, we did not find any such associations with available metadata when examining samples individually (Fig. 5). Color-coding by patient or sample type revealed no obvious trend (Fig. 5a), while colorcoding by sequencing center reveals that Mycobacterium reads are associated predominantly with just one sequencing center (Fig. 5b).

In STAD, there appears to be no difference in bacterial abundances or ratios correlated with sequencing center, collection center, or plate when samples are examined individually (Fig. 6). The samples have a unimodal distribution for all of the comparisons, except for the comparisons with samples containing Pseudomonas reads where a small subset of samples collected from Asterand have an increased abundance of Pseudomonas read pairs, therefore separating them from the other samples. A more precise attribute to distinguish these samples from the others could not be determined. This indicates that there is little variation within the single sequencing center, collection center, and plate from which these samples were collected. As the plot of all the cancer types illustrated, there is very little variation for the comparison of Staphylococcus read pairs to Propionibacterium read pairs (Fig. 3), reinforcing that these two species are likely co-contaminants. Therefore, we conclude that Pseudomonas read pairs result from either separate contamination for which a source could not be identified or from a biologically relevant source like the stomach adenocarcinoma microbiome. 


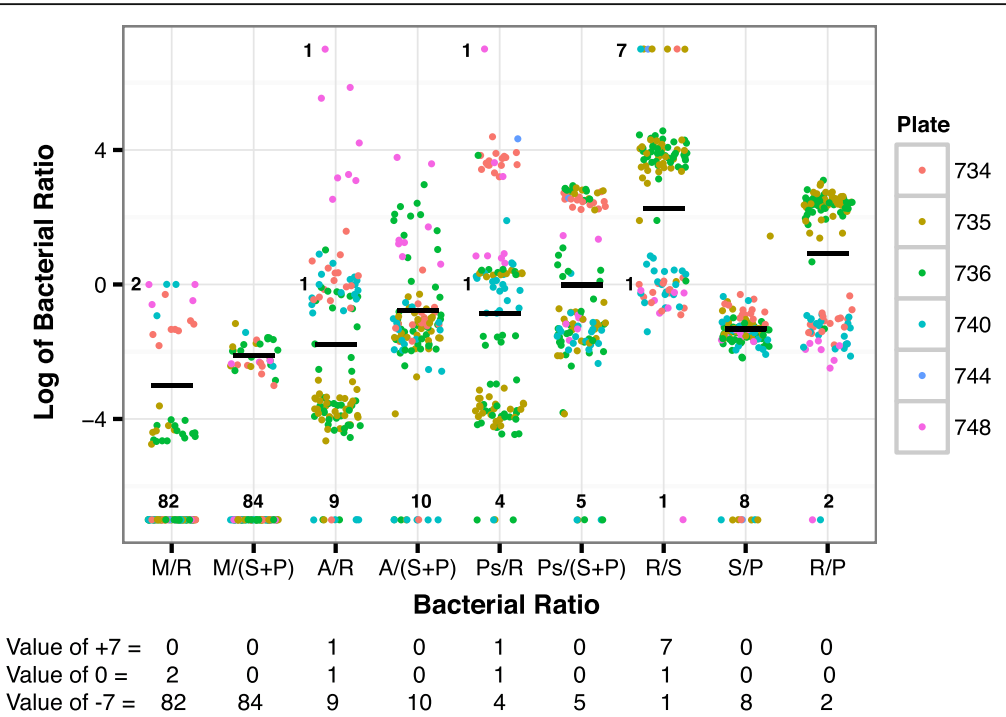

Fig. 4 The $\log _{10}$-transformed ratios of bacterial read pairs from a specific taxon to the total number of bacterial read pairs from that patient were calculated for all AML samples as described in Fig. 3. Ratios where the numerator was 0 were assigned an arbitrary value of -7 , ratios where both the numerator and denominator were 0 were assigned an arbitrary value of 0 , and comparisons where the denominator was 0 were assigned an arbitrary value of 7 . Counts for the number of patients where one of these arbitrary values were assigned are shown below the $x$-axis. When $>5$ points are at a given value, some data points may overlap each other making it impossible for all data points to be seen. The samples were all collected and sequenced at the same locations, and the data are color-coded by plate. The samples on plate 735 and 736 have a higher abundance of Ralstonia spp., suggesting that these samples may have been contaminated and this may be responsible for the bimodal distribution of samples in those comparisons. As was seen with the plot of all the cancer types, there is very little variation for the comparison of Staphylococcus spp. to Propionibacterium spp

To determine if removal of these contaminants from our dataset would reduce bacterial diversity within each cancer type, alpha diversity was calculated before and after applying a filter to remove the contamination identified above. For filtering, all S. epidermidis and Propionibacterium read pairs were removed. Additionally, Mycobacterium read pairs were removed from the OV and GBM datasets and Ralstonia read pairs were removed from the AML dataset. After subsampling all cancer types to the minimum number of reads in the smallest cancer type, the numbers of OTUs were counted (Additional file 6: Figure S5). KIRC had the highest amount of diversity across the cancer types, while AML had the lowest amount of diversity (Additional file 6: Figure S5). It does not appear that filtering for these potential contaminants substantially altered the alpha diversity of any cancer type (Additional file 6: Figure S5).

\section{Discussion}

Lack of evidence supporting contamination as origin of some dominant bacterial species

Through this comprehensive evaluation of the bacterial taxa present in the Illumina paired-end reads from an early release of TCGA data, we describe the presence of bacteria associated with specific cancer types. Many researchers suggest removing reads from commonly contaminating taxa. However, this poses numerous problems, most notably that you introduce a bias. It also assumes that contamination sources are static, when they are actually fluid. For both technical and practical reasons, there is no negative control for sequencing. With this in mind, all of the data were processed and analyzed in the same manner. However, we recognize that the GBM samples can serve as a quasi-negative control since a brain tumor is unlikely to have resident bacteria due to the blood-brain barrier. The only bacterial reads found to be substantially associated with GBM samples were of Mycobacterium origin, which was deemed to be a sequence center-level contamination issue.

Upon conclusion of the various tests and proxies used to examine bacterial contamination of the samples, the presence of Acinetobacter and Pseudomonas spp. in AML and Pseudomonas spp. in STAD could not be attributed to contamination. Regardless of the measures taken to associate metadata with these bacteria, no associations between the collection centers, plates, sequencing centers, etc. were found. We also examined the sequencing projects that occurred at the British Columbia Genome Sequencing Center (BCGSC), which sequenced the AML and STAD samples. We could not find Pseudomonas spp. or Acinetobacter spp. sequencing projects ongoing at the approximate time the samples may have been sequenced, and we did not find many bacterial sequencing projects there, in general. This lends support to the Pseudomonas-like and Acinetobacter-like sequencing reads being biologically relevant. In 


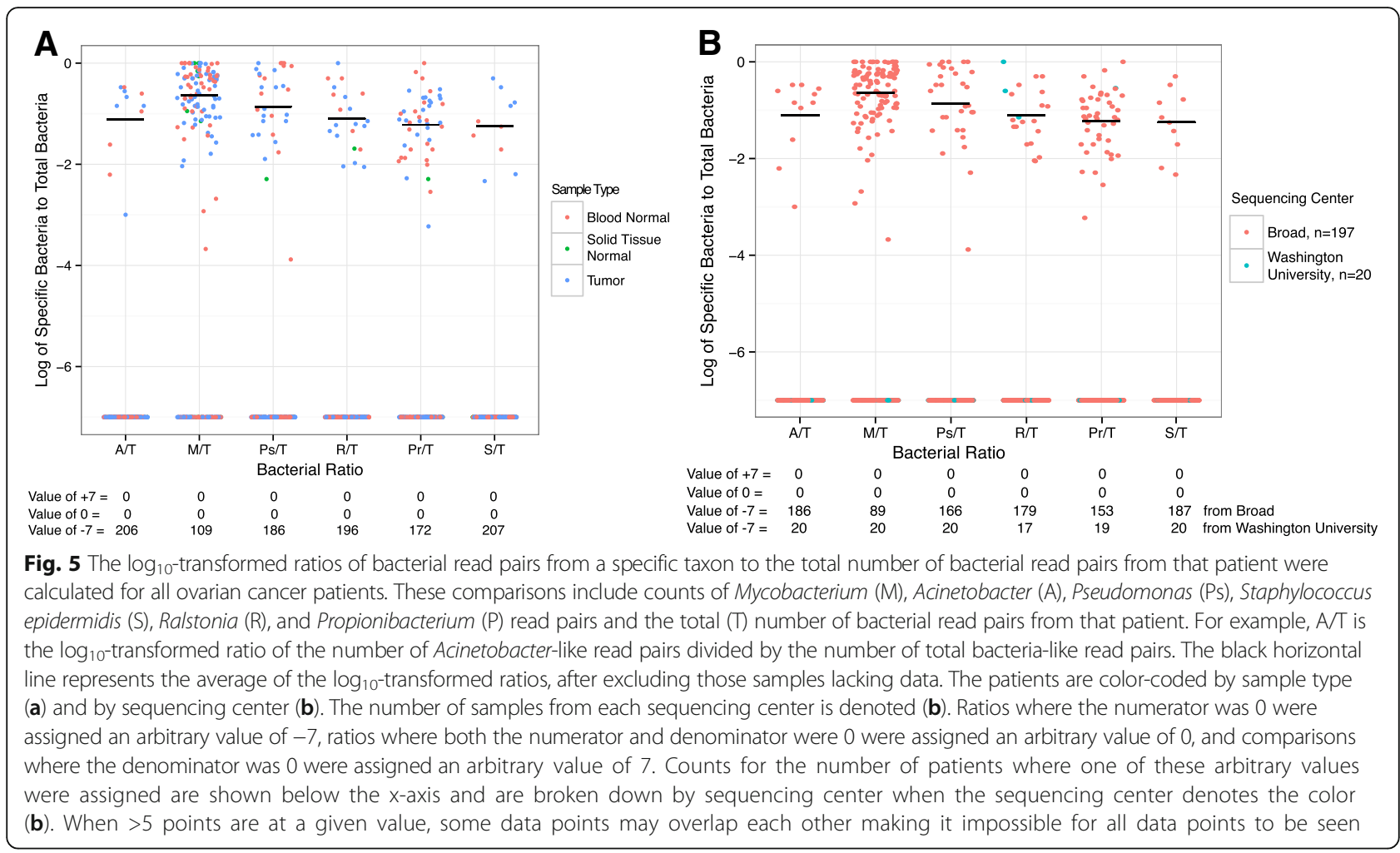

the STAD dataset, samples were collected from two different collection centers, and while there was no correlation between the bacteria present and collection centers for most samples, a small subset of samples from Asterand had an increased abundance of Pseudomonas-like read pairs. As AML and STAD samples were only sequenced at BCGSC in this release of TCGA data, no further conclusions based on sequencing center could be made.

However, there are factors that complicate these analyses. Experimental methods for extracting DNA and RNA can confound these results, as these samples were collected for the primary purpose of analyzing the cancer genome and not studying bacteria. Some DNA extraction methods are preferred for breaking bacterial cell walls, meaning that samples undergoing methods that are more efficient at breaking the bacterial cell wall, such as bead beating, could have a higher bacterial yield than those extracted with other methods. Another complication of this analysis is that all of the AML samples were collected from a single center and sequenced at a single center. Therefore, there were no samples from an alternate collection center or sequencing center to use as a comparison, at least in this release of TCGA data. Additionally, the AML and STAD datasets were poly-A-selected RNA-Seq data, which will impact the bacteria identified by these analyses as discussed in the "Background" section. One might assume that this poly-A-selected RNA-Seq data is devoid of bacterial mRNA; however, we have observed in multiple projects that this is not the case. In terms of identifying cancer-related bacteria in poly-A sequencing, we only identified $142 \mathrm{H}$. pylori read pairs across the entire TCGA dataset (MicroView). However, we have been able to identify many more read pairs attributed to $H$. pylori in other poly-A-selected transcriptomics data generated by our group (data not shown). Therefore, in the case of TCGA data, we believe the lack of $H$. pylori read pairs was not caused by poly-A selection, but other factors (e.g., samples being collected from individuals who did not have an active $H$. pylori infection). An additional example of identifying bacteria in poly-A-selected data is a project on the fruit fly Drosophila ananassae and its Wolbachia endosymbiont (SRA Project SRP061993). A total RNA library contained 193,612 Wolbachia reads and 8,889,348 fruit fly reads that mapped while a poly-A-enriched RNA library contained 1923 Wolbachia reads and 9,318,954 fruit fly reads that mapped. This indicates that poly-Aselected RNA libraries contain bacterial reads that can be used to examine the microbiome. However, it is likely that they do not provide a complete, unbiased picture of the bacterial transcripts present in the sample.

\section{Pitfalls of explorations of public sequence data}

The 2003 Fort Lauderdale agreement on "Sharing Data from Large-scale Biological Research Projects" and it predecessor, the 1996 Bermuda Principles, laid out foundations for the sharing of biological data, most notably 

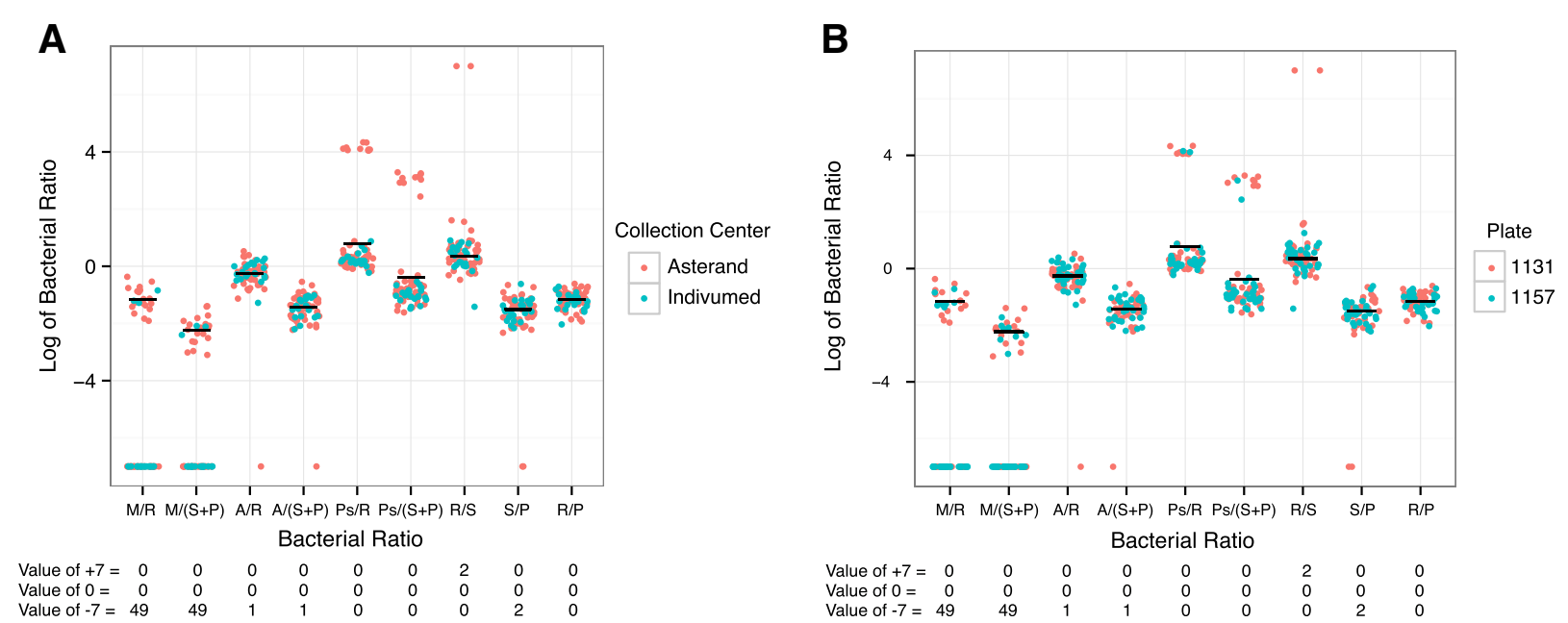

Fig. 6 The $\log _{10}$-transformed ratios of bacterial counts by patient were calculated as described in Fig. 3 for stomach adenocarcinoma samples with the various panels color-coded by collection center (a) and plate (b). For each bacterial comparison, there is a mostly unimodal distribution indicating that there is little variation across samples. The ratios with Pseudomonas spp. have a few outliers from the Asterand collection center, but otherwise, no correlation between these factors and the relative abundance of the specific bacteria can be identified

genomics data. Combined with ever-evolving policies from funding agencies that support these data sharing ideals, vast amounts of data are publicly available for secondary uses. While data is frequently deposited, it is less frequently used for such secondary analyses. Our group uses such data to identify previously undetected interdomain lateral gene transfers [30,37], while other notable examples of secondary data use involve studies on identifying biomarkers for preeclampsia [38], cell surface targets of medulloblastoma [39], and factors associated with organ transplant rejection [40, 41]. When possible, it is best to merge multiple datasets and find characteristics in common across those datasets, lending support to those conclusions. Here, we use such common results to identify common contaminants in some datasets, like staphylococci and propionibacteria, which are frequently found across all datasets and in a similar relative proportion to one another.

While such cross dataset analysis is possible when numerous datasets can be aggregated, it is not always possible. In such cases where a limited number of datasets of a given type are available, it can be informative to evaluate potential batch effects or other correlations that can be linked via the metadata. In the case of our analysis, this revealed a correlation between sequencing center and M. tuberculosis complex in the OV and GBM samples and a correlation between plates and Ralstonia spp. in AML samples.

It is also important to recognize the limitations of such secondary data analyses, especially when data is used to address an alternate question. For example, much of the TCGA data is RNA-Seq or whole exome data collected following selective capture of certain nucleic acids such that bacterial profiles examined may be incomplete. A recent study showed that microbial communities in the saliva were not biased in exome sequence data compared to whole genome sequence data [42]. However, it is not clear how widespread this observation may be, and while some bacterial associations can be identified, others may be lost due to the methods used. In the release of the TCGA that we used, there were no samples that had whole genome sequencing (WGS), whole exome sequencing (WXS), and RNA-Seq data. One GBM sample had DNA and RNA extracted, and both sequence types possessed bacterial read pairs. The RNA data had 208 bacterial read pairs, primarily comprised of Enterobacteriaceae. The DNA sample contained 1421 bacterial read pairs with one third of the read pairs contributed by $P$. acnes. Both of these bacteria we attribute as contamination, and otherwise, the bacterial taxa identified were very similar. This is not surprising given that GBM samples are not expected to have bacterial sequences present, as noted above. We also compared one STAD RNA-Seq sample in this dataset with WGS and WXS data from the same sample from a subsequent TCGA data release. While there were a few minor differences between WGS and WXS, the bacterial taxa identified were very similar between the two sequencing types (Additional file 7: Figure S6). There were almost equal proportions of Firmicutes, Fusobacteria, and Bacteroidetes when compared between the WGS and WXS results. The specificity of the taxonomic assignments was also very similar between WGS and WXS. However, there were large differences in bacterial diversity when 
comparing RNA-Seq data to WGS or WXS, with RNASeq data having an enrichment of Proteobacteria and lacking a significant number of read pairs from other phyla (Additional file 7: Figure S6). While the WGS and WXS data also contained bacterial read pairs from Proteobacteria, these read pairs were in the minority (Additional file 7: Figure S6).

There may also be a difference in the types of genes found from each bacterial taxa (Additional file 8: Table S1). GBM and OV were the only cancer types investigated where DNA sequencing was completed, which is reflected by the increased percentage of GBM read pairs that aligned to protein coding regions (CDSs) compared to the other cancer types. The other eight cancer types, which were RNA-Seq datasets, had $68-90 \%$ of the bacterial read pairs aligning to rRNA, with only $3-31 \%$ of the reads aligning to coding sequences (Additional file 8: Table S1). This likely reflects the normal composition of these nucleic acids, with total RNA dominated by rRNA while DNA has a composition reflecting the entire genome.

In the cases of the Acinetobacter association with AML and the Pseudomonas association with STAD, we cannot distinguish between contamination and a biologically relevant association. Since samples are not available for follow-up studies, prospective samples will need to be collected to test these hypotheses in the future. Despite the need for further experimentation, analyses of large public data repositories such as this one, provide an important and cost-effective opportunity to develop numerous new hypotheses that have the potential to challenge dogma.

\section{Conclusions}

A more thorough evaluation of publicly available sequence data with a microbiome-focused analysis may be fruitful. However, methods to examine contamination and batch effects should be used. In the case of public sequencing data, it can sometimes be very difficult to determine experimental protocol and determine what bacterial taxa in the samples may be due to batch effects from experimental protocols and what bacteria are actually in the samples. This suggests that more metadata, as well as better structured metadata, may be necessary to ensure these datasets can be used more successfully in secondary analyses. Based on our analysis, we suggest that nucleic acid extraction method, collection site, sequencing site, tissue type, sequence type, library type, library methods, and antibiotic treatment must be included in a manner that is easy to retrieve. In addition, researchers acutely need methods to track other nucleicacid-based projects simultaneously occurring in the source sites, including the tissue source site, the sequencing center, and any other collection sites.

\section{Methods}

\section{Pipeline for identifying bacterial reads and taxonomic} assignments

The pipeline for executing alignments with BWA version 0.5.9-r16 and discarding low complexity and duplicate reads with PRINSEQ version 0.20 .3 [43] has previously been described [30]. Briefly, alignments were constructed to all complete bacterial genomes in RefSeq as well as the human reference genome, hg19, using BWA ALN version 0.5.9-r16 [44]. Other alignment algorithms including BWA MEM and MOSAIK were tested on other datasets. BWA MEM is more sensitive, but less specific, unless the database includes the host and all microbes, which was not possible here.

After aligning the read pairs to all of the references, pairs of reads where neither read matched hg19, but both matched bacteria were selected for further analysis. OTUs were calculated for each read pair using the BWA results $[30,45]$. Each individual read in the read pair was given the taxonomic assignment of its best match, or the aggregate common taxonomy of all of its best matches. The read pair was then assigned an OTU by comparing the two taxonomic assignments of the reads in the pair and using the common taxonomy (Additional file 1: Figure S1). Reads were attributed to the bacterial portion of the microbiome when both reads had an OTU suggesting "bacteria."

\section{Construction of MicroView interactive data browser}

Taxonomic assignments and metadata for approximately 36 million de-identified reads were loaded into a PostgreSQL relational database. Materialized views were then created to summarize read counts by every observed combination of metadata field values and taxonomic assignment. Components of the LGTView user interface [30] were combined with a new set of Python CGI scripts to create MicroView, an interactive interface that allows a user to drill down into the 36 million reads and 9 cancer types using any combination of the available metadata fields: cancer type, plate, sequencing center, tissue source site, SRA run ID, sample/vial, analyte, taxonomic assignment, and anonymized sample id.

\section{Heat map preparation}

The 36 million putative microbiome reads were parsed into their most specific taxonomic category to the family level with the exception of reads classified as arising from Ralstonia, Mycobacterium, Propionibacterium, Staphylococcus, Acinetobacter, or Pseudomonas, where a genus level assignment was used. The $\log _{10}$-transformed read counts per sample per taxonomic category were normalized to the number of total reads per sample, multiplied by 100,000 and plotted in a heat map (Fig. 2). A sample 
was defined as a specific nucleic acid sequenced by a specific center. Therefore, DNA and RNA would be considered different samples as would the same DNA that was sequenced by two different sequencing centers. Only taxa with $>20$ reads per one million total reads are visualized. Samples were clustered using agglomerative hierarchical clustering in the heatmap. 2 function of GPLOTS version 3.0.1 running in $\mathrm{R}$ version 3.3.1. Each sample was colorcoded by the associated metadata, which is organized in the key by the numeric code assigned by the TCGA.

\section{BLASTN searches for Mycobacterium}

Searches to confirm $M$. tuberculosis complex read pairs were completed using the NCBI tool BLASTN [46] with the default settings. All of the OV and GBM read pairs with matches identified by BWA to $M$. tuberculosis complex were searched against the NT database. Figures representing the bacterial proportions in cancer and normal samples were created using Krona [35].

\section{Log ratio calculations comparing Mycobacterium read pairs to total read pairs}

The $\log _{10}$ ratio comparing the number of Mycobacterium spp. read pairs per run to the total number of reads per run was calculated as the $\log _{10}$ of the number of Mycobacterium spp. read pairs in each run divided by the total number of read pairs in each run. The ratio comparing the number of Mycobacterium spp. read pairs per sample to the total number of read pairs per sample was calculated as the $\log _{10}$ of the number of Mycobacterium spp. read pairs in each sample divided by the total number of read pairs in each sample.

\section{Log ratio calculations comparing select bacterial read pairs}

Mycobacterium (M), Acinetobacter (A), and Pseudomonas (Ps) read pairs were defined as microbiomeassociated bacteria. S. epidermidis (S), Ralstonia (R), and Propionibacterium (P) read pairs were defined as potential contaminant bacteria. The $\log _{10}$ ratios comparing the microbiome-associated bacteria to the potential contaminant bacteria were calculated by taking the $\log _{10}$ (number of microbiome-associated bacteria read pairs/ number of contaminant bacteria read pairs). Subsequently, S. epidermidis and Propionibacterium $(\mathrm{S}+\mathrm{P})$ were grouped together after an initial comparison found them to frequently have the same relative proportions. They are both commonly found on the human skin and may arrive in the samples in the same manner. The average of each $\log _{10}$-transformed ratio across all the cancer types or across all of the samples was calculated and illustrated by a black horizontal line. As not all of the samples had read pairs with a particular bacterial OTU, some of the comparisons would not be calculated. Ratios where the numerator was 0 were assigned an arbitrary value of -7 for that ratio, ratios where both the numerator and denominator were 0 were assigned an arbitrary value of 0 , and ratios where the denominator was 0 were assigned an arbitrary value of 7 .

\section{Alpha diversity calculations}

Alpha diversity was calculated by counting the total number of OTUs present from each cancer type before and after filtering for potential contaminants. Due to the large discrepancies in the number of bacterial read pairs across all of the cancer types, subsampling was necessary. Therefore, all cancer types were subsampled to the number of bacterial read pairs from GBM, which had the fewest bacterial read pairs. Subsampling was done with Mothur [47] version 1.36.1, using the sub.sample function with default settings. From this, the total number of OTUs present within each cancer type were counted and plotted. In the filtered dataset, all S. epidermidis and Propionibacterium read pairs were removed. In addition, all Ralstonia read pairs were removed from the AML dataset and all Mycobacterium read pairs were removed from the OV and GBM datasets.

\section{Gene feature calculations}

The mappings of each bacterial read pair to all of the complete bacterial genomes in RefSeq were combined with genome annotations to determine the gene features for each bacterial read pair alignment.

\section{Additional files}

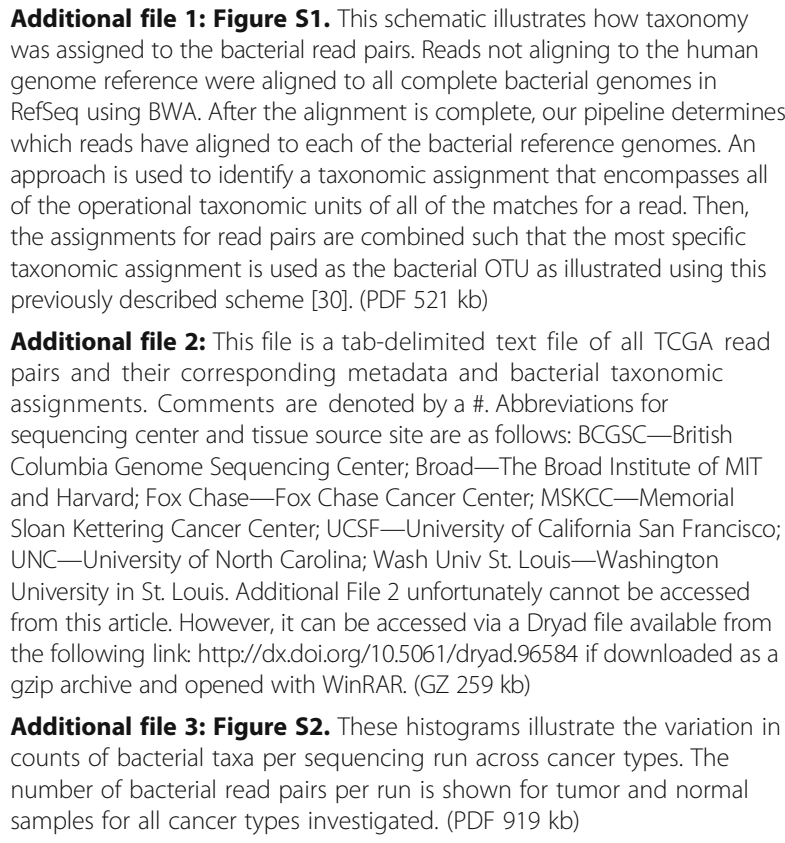
was assigned to the bacterial read pairs. Reads not aligning to the human genome reference were aligned to all complete bacterial genomes in RefSeq using BWA. After the alignment is complete, our pipeline determines which reads have aligned to each of the bacterial reference genomes. An approach is used to identify a taxonomic assignment that encompasses all of the operational taxonomic units of all of the matches for a read. Then, the assignments for read pairs are combined such that the most specific taxonomic assignment is used as the bacterial OTU as illustrated using this previously described scheme [30]. (PDF $521 \mathrm{~kb}$ )

Additional file 2: This file is a tab-delimited text file of all TCGA read pairs and their corresponding metadata and bacterial taxonomic assignments. Comments are denoted by a \#. Abbreviations for sequencing center and tissue source site are as follows: BCGSC_British Columbia Genome Sequencing Center; Broad_-The Broad Institute of MIT and Harvard; Fox Chase_-Fox Chase Cancer Center; MSKCC_-Memorial Sloan Kettering Cancer Center; UCSF-University of California San Francisco; UNC - University of North Carolina; Wash Univ St. Louis-Washington University in St. Louis. Additional File 2 unfortunately cannot be accessed from this article. However, it can be accessed via a Dryad file available from the following link: http://dx.doi.org/10.5061/dryad.96584 if downloaded as a gzip archive and opened with WinRAR. (GZ 259 kb)

Additional file 3: Figure S2. These histograms illustrate the variation in counts of bacterial taxa per sequencing run across cancer types. The number of bacterial read pairs per run is shown for tumor and normal samples for all cancer types investigated. (PDF 919 kb) 
Additional file 4: Figure S3. The proportions of bacterial read pairs for both tumor (panel A) and normal-matched tissue (panel B) for glioblastoma multiforme (GBM) are illustrated. The GBM dataset had about the same number of bacterial read pairs per sample for both the 78 normal and the 78 tumor samples. Both sample types had about a third of the read pairs from Ralstonia picketti with low levels of read pairs from Enterobacteriaceae, Pseudomonas aeruginosa, and Mycobacterium tuberculosis complex. (PDF $841 \mathrm{~kb}$ )

Additional file 5: Figure S4. The $\log _{10}$-transformed ratios of bacterial counts by patient were calculated for the breast cancer data as described in Fig. 3. Samples were color-coded by sample type in panel $A$ and by plate in panel B. All of the samples were sequenced at the same center. In panel B, the samples segregate by plate for ratios containing Ralstonia spp., suggesting that these plates may have been contaminated. (PDF $329 \mathrm{~kb}$ )

Additional file 6: Figure S5. Alpha diversity for all cancer types was compared pre- and post-filtering. The total number of OTUs before and after filtering for potential contaminant bacterial read pairs is plotted for each cancer type. Subsampling was done to accurately compare the large datasets, like AML and STAD to the smallest dataset, GBM. No major differences in alpha diversity were observed after filtering. (PDF $118 \mathrm{~kb}$ )

Additional file 7: Figure S6. The proportion of bacterial read pairs for RNA-Seq (panel A), whole genome sequencing (WGS, panel B), and whole exome sequencing (WXS, panel C) for an individual stomach adenocarcinoma (STAD) sample are illustrated. The RNA-Seq data shows an enrichment of Proteobacteria that have low proportions in the WGS and WXS data. WGS and WXS sequencing methods resulted in a more diverse collection of bacterial read pairs with only minor differences between the two methods. (PDF $445 \mathrm{~kb}$ )

Additional file 8: Table S1. Types of gene features represented by the bacterial read pairs of each cancer type. (DOC $54 \mathrm{~kb}$ )

\section{Abbreviations}

AML: Acute myeloid leukemia; BCGSC: British Columbia Genome Sequencing Center; BRCA: Breast cancer; Broad: The Broad Institute of MIT and Harvard; BWA: Burrows-Wheeler aligner; EBV: Epstein-Barr virus; Fox Chase: Fox Chase Cancer Center; GBM: Glioblastoma multiforme; HBV: Hepatitis B virus; HIV: Human immunodeficiency virus; HPV: Human papillomavirus; KIRC: Kidney clear cell carcinoma; KIRP: Kidney papillary carcinoma; LGT: Lateral gene transfer; LUAD: Lung adenocarcinoma; LUSC: Lung squamous cell carcinoma; MALT: Mucosa-associated lymphoid tissue; MSKCC: Memorial Sloan Kettering Cancer Center; OTU: Operational taxonomic unit; OV: Ovarian serous cystadenocarcinoma; RNA-Seq: RNA sequencing; SRA: Sequence Read Archive; STAD: Stomach adenocarcinoma; TCGA: The Cancer Genome Atlas; UCSF: University of California San Francisco; UNC: University of North Carolina; Wash Univ St. Louis: Washington University in St. Louis; WGA: Whole genome amplified; WGS: Whole genome sequencing; WXS: Whole exome sequencing

\section{Acknowledgements}

We thank Dr. Johanna B. Holm for her thoughtful discussion of the ideal methods for calculating alpha diversity in this novel framework. The results published here are in part based upon data generated by The Cancer Genome Atlas (TCGA) project established by the NCl and NHGRI. Information about TCGA and the investigators and institutions which constitute the TCGA research network can be found at "http://cancergenome.nih.gov". Initial sequence alignments were conducted on the National Science Foundation funded Data Intensive Grid (DIAG; www.diagcomputing.org) through MRI-R2 project \#DBI-0959894.

\section{Funding}

This work was funded by the National Institutes of Health through the $\mathrm{NIH}$ Director's New Innovator Award Program (1-DP2-OD007372) and an NIH Director's Transformative Research Award (1-R01-CA206188).

\section{Availability of data and materials}

The datasets analyzed during the current study were available from TCGA (http://cancergenome.nih.gov/) in the Sequence Read Archive through dbGap as phs000178.v4.p4. All data generated during the current study are included in this published article and its supplementary and additional files.
The Dryad accession number for Additional file 2 is doi:10.5061/dryad.96584 and can be accessed at: http://dx.doi.org/10.5061/dryad.96584.

\section{Authors' contributions}

KMR drafted the manuscript, developed the pipeline, analyzed the data, and provided guidance to KEA. JC implemented the MicroView instance for interacting with the data. JSAM created the heat map showing the bacterial taxa present in each sample. KEA conducted the comparison of the microbiome composition of transcriptome, exome, and whole genome sequencing data from a single sample. JCDH edited the manuscript, provided guidance on pipeline development and analysis of data, and oversaw the project. All authors read and approved the final manuscript.

\section{Competing interests}

The authors declare that they have no competing interests.

\section{Consent for publication}

Not applicable.

\section{Ethics approval and consent to participate}

Not applicable.

\section{Author details}

${ }^{1}$ Institute for Genome Sciences, University of Maryland School of Medicine, Baltimore, MD, USA. ${ }^{2}$ Department of Microbiology and Immunology, University of Maryland School of Medicine, Baltimore, MD, USA.

${ }^{3}$ Greenebaum Cancer Center, University of Maryland School of Medicine, Baltimore, MD, USA.

Received: 1 September 2016 Accepted: 15 December 2016

Published online: 25 January 2017

\section{References}

1. de Martel C, Ferlay J, Franceschi S, Vignat J, Bray F, Forman D, et al. Global burden of cancers attributable to infections in 2008: a review and synthetic analysis. Lancet Oncol. 2012;13(6):607-15. doi:10.1016/ s1470-2045(12)70137-7.

2. Sarid R, Gao SJ. Viruses and human cancer: from detection to causality. Cancer Lett. 2011;305(2):218-27. doi:10.1016/..canlet.2010.09.011.

3. Van Tine BA, Kappes JC, Banerjee NS, Knops J, Lai L, Steenbergen RD, et al. Clonal selection for transcriptionally active viral oncogenes during progression to cancer. J Virol. 2004;78(20):11172-86. doi:10.1128/jvi.78.20. 11172-11186.2004

4. Robinson KM, Dunning Hotopp JC. Mobile elements and viral integrations prompt considerations for bacterial DNA integration as a novel carcinogen. Cancer Lett. 2014. doi:10.1016/..canlet.2014.05.021.

5. Peek Jr RM, Blaser MJ. Helicobacter pylori and gastrointestinal tract adenocarcinomas. Nat Rev Cancer. 2002;2(1):28-37. doi:10.1038/nrc703.

6. Vogtmann E, Goedert JJ. Epidemiologic studies of the human microbiome and cancer. Br J Cancer. 2016;114(3):237-42. doi:10.1038/bjc.2015.465.

7. Fried B, Reddy A, Mayer D. Helminths in human carcinogenesis. Cancer Lett. 2011;305(2):239-49. doi:10.1016/j.canlet.2010.07.008.

8. Dutta U, Garg PK, Kumar R, Tandon RK. Typhoid carriers among patients with gallstones are at increased risk for carcinoma of the gallbladder. Am J Gastroenterol. 2000;95(3):784-7. doi:10.1111/j.1572-0241.2000.01860.x.

9. Littman AJ, White E, Jackson LA, Thornquist MD, Gaydos CA, Goodman GE, et al. Chlamydia pneumoniae infection and risk of lung cancer. Cancer Epidemiol Biomarkers Prev. 2004;13(10):1624-30.

10. Sears CL, Pardoll DM. Perspective: alpha-bugs, their microbial partners, and the link to colon cancer. J Infect Dis. 2011;203(3):306-11. doi:10.1093/jinfdis/jiq061.

11. Sears CL. Enterotoxigenic Bacteroides fragilis: a rogue among symbiotes. Clin Microbiol Rev. 2009;22(2):349-69. doi:10.1128/cmr.00053-08.

12. Biarc J, Nguyen IS, Pini A, Gosse F, Richert S, Thierse D, et al. Carcinogenic properties of proteins with pro-inflammatory activity from Streptococcus infantarius (formerly S. bovis). Carcinogenesis. 2004;25(8):1477-84. doi:10.1093/carcin/bgh091.

13. Martin HM, Campbell BJ, Hart CA, Mpofu C, Nayar M, Singh R, et al. Enhanced Escherichia coli adherence and invasion in Crohn's disease and colon cancer. Gastroenterol. 2004;127(1):80-93. 
14. Kostic AD, Gevers D, Pedamallu CS, Michaud M, Duke F, Earl AM, et al. Genomic analysis identifies association of Fusobacterium with colorectal carcinoma. Genome Res. 2012;22(2):292-8. doi:10.1101/gr.126573.111.

15. Castellarin M, Warren RL, Freeman JD, Dreolini L, Krzywinski M, Strauss J, et al. Fusobacterium nucleatum infection is prevalent in human colorectal carcinoma. Genome Res. 2012;22(2):299-306. doi:10.1101/gr.126516.111.

16. Rubinstein MR, Wang X, Liu W, Hao Y, Cai G, Han YW. Fusobacterium nucleatum promotes colorectal carcinogenesis by modulating E-cadherin/ beta-catenin signaling via its FadA adhesin. Cell Host Microbe. 2013;14(2): 195-206. doi:10.1016/j.chom.2013.07.012.

17. Kostic AD, Chun E, Robertson L, Glickman JN, Gallini CA, Michaud M, et al. Fusobacterium nucleatum potentiates intestinal tumorigenesis and modulates the tumor-immune microenvironment. Cell Host Microbe. 2013; 14(2):207-15. doi:10.1016/j.chom.2013.07.007.

18. Marchesi JR, Dutilh BE, Hall N, Peters WH, Roelofs R, Boleij A, et al. Towards the human colorectal cancer microbiome. PLoS One. 2011;6(5), e20447. doi:10.1371/journal.pone.0020447.

19. Lax AJ, Thomas W. How bacteria could cause cancer: one step at a time Trends Microbiol. 2002;10(6):293-9.

20. Botelho MC, Machado JC, Brindley PJ, Correia da Costa JM. Targeting molecular signaling pathways of Schistosoma haemotobium infection in bladder cancer. Virulence. 2011;2(4):267-79.

21. Ferguson AR. Associated bilharziosis and primary malignant disease of the urinary bladder, with observations on a series of forty cases. J Pathol. 1911; 16(1):76-94. doi:10.1002/path.1700160107.

22. Badawi AF, Mostafa MH, Probert A, O'Connor PJ. Role of schistosomiasis in human bladder cancer: evidence of association, aetiological factors, and basic mechanisms of carcinogenesis. Eur J Cancer Prev. 1995;4(1):45-59.

23. Botelho M, Ferreira AC, Oliveira MJ, Domingues A, Machado JC, da Costa JM. Schistosoma haematobium total antigen induces increased proliferation, migration and invasion, and decreases apoptosis of normal epithelial cells. Int J Parasitol. 2009;39(10):1083-91. doi:10.1016/j.ijpara.2009.02.016.

24. Rosin MP, Anwar WA, Ward AJ. Inflammation, chromosomal instability, and cancer: the schistosomiasis model. Cancer Res. 1994;54(7 Suppl):1929s-33.

25. Kim SS, Ruiz VE, Carroll JD, Moss SF. Helicobacter pylori in the pathogenesis of gastric cancer and gastric lymphoma. Cancer Lett. 2011;305(2):228-38. doi:10.1016/j.canlet.2010.07.014.

26. Chan AO, Peng JZ, Lam SK, Lai KC, Yuen MF, Cheung HK, et al. Eradication of Helicobacter pylori infection reverses E-cadherin promoter hypermethylation. Gut. 2006:55(4):463-8. doi:10.1136/gut.2005.077776.

27. Hatakeyama M. Oncogenic mechanisms of the Helicobacter pylori CagA protein. Nat Rev Cancer. 2004;4(9):688-94. doi:10.1038/nrc1433.

28. Kostic AD, Ojesina Al, Pedamallu CS, Jung J, Verhaak RG, Getz G, et al. PathSeq: software to identify or discover microbes by deep sequencing of human tissue. Nat Biotechnol. 2011;29(5):393-6. doi:10.1038/nbt.1868.

29. The Cancer Genome Atlas Research N. Comprehensive molecular characterization of gastric adenocarcinoma. Nature. 2014;513(7517):202-9. doi:10.1038/nature13480

30. Riley DR, Sieber KB, Robinson KM, White JR, Ganesan A, Nourbakhsh S, et al. Bacteria-human somatic cell lateral gene transfer is enriched in cancer samples. PLoS Comput Biol. 2013;9(6), e1003107. doi:10.1371/journal.pcbi. 1003107.

31. Salter SJ, Cox MJ, Turek EM, Calus ST, Cookson WO, Moffatt MF, et al. Reagent and laboratory contamination can critically impact sequence-based microbiome analyses. BMC Biol. 2014;12:87. doi:10.1186/s12915-014-0087-z.

32. Naccache $S N$, Greninger AL, Lee $D$, Coffey LL, Phan T, Rein-Weston A, et al. The perils of pathogen discovery: origin of a novel parvovirus-like hybrid genome traced to nucleic acid extraction spin columns. J Virol. 2013;87(22): 11966-77. doi:10.1128/jvi.02323-13.

33. Cantalupo PG, Katz JP, Pipas JM. HeLa nucleic acid contamination in the Cancer Genome Atlas leads to the misidentification of human papillomavirus 18. J Virol. 2015;89(8):4051-7. doi:10.1128/jvi.03365-14.

34. Tang K-W, Alaei-Mahabadi B, Samuelsson T, Lindh M, Larsson E. The landscape of viral expression and host gene fusion and adaptation in human cancer. Nat Commun. 2013;4:2513. doi:10.1038/ncomms3513.

35. Ondov BD, Bergman NH, Phillippy AM. Interactive metagenomic visualization in a web browser. BMC Bioinformatics. 2011;12:385. doi:10.1186/1471-2105-12-385.

36. Schwabe RF, Jobin C. The microbiome and cancer. Nat Rev Cancer. 2013; 13(11):800-12. doi:10.1038/nrc3610.
37. Dunning Hotopp JC, Clark ME, Oliveira DC, Foster JM, Fischer P, Munoz Torres MC, et al. Widespread lateral gene transfer from intracellular bacteria to multicellular eukaryotes. Science. 2007;317(5845):1753-6. doi:10.1126/ science.1142490.

38. Liu LY, Yang T, Ji J, Wen Q, Morgan AA, Jin B, et al. Integrating multiple 'omics' analyses identifies serological protein biomarkers for preeclampsia. BMC Med. 2013;11:236. doi:10.1186/1741-7015-11-236.

39. Haeberle H, Dudley JT, Liu JT, Butte AJ, Contag CH. Identification of cell surface targets through meta-analysis of microarray data. Neoplasia. 2012; 14(7):666-9.

40. Morgan AA, Khatri P, Jones RH, Sarwal MM, Butte AJ. Comparison of multiplex meta analysis techniques for understanding the acute rejection of solid organ transplants. BMC Bioinform. 2010;11 Suppl 9:S6. doi:10.1186/ 1471-2105-11-s9-s6.

41. Chen R, Sigdel TK, Li L, Kambham N, Dudley JT, Hsieh SC, et al. Differentially expressed RNA from public microarray data identifies serum protein biomarkers for cross-organ transplant rejection and other conditions. PLoS Comput Biol. 2010;6(9). doi:10.1371/journal.pcbi. 1000940.

42. Kidd JM, Sharpton TJ, Bobo D, Norman PJ, Martin AR, Carpenter ML, et al. Exome capture from saliva produces high quality genomic and metagenomic data. BMC Genomics. 2014;15:262. doi:10.1186/1471-2164-15-262.

43. Schmieder R, Edwards R. Quality control and preprocessing of metagenomic datasets. Bioinformatics. 2011;27(6):863-4. doi:10.1093/ bioinformatics/btr026.

44. Li H, Durbin R. Fast and accurate short read alignment with BurrowsWheeler transform. Bioinformatics. 2009:25(14):1754-60. doi:10.1093/ bioinformatics/btp324.

45. Huson DH, Auch AF, Qi J, Schuster SC. MEGAN analysis of metagenomic data. Genome Res. 2007;17(3):377-86. doi:10.1101/gr.5969107.

46. Altschul SF, Gish W, Miller W, Myers EW, Lipman DJ. Basic local alignment search tool. J Mol Biol. 1990;215(3):403-10. doi:10.1016/s00222836(05)80360-2.

47. Schloss PD, Westcott SL, Ryabin T, Hall JR, Hartmann M, Hollister EB, et al. Introducing mothur: open-source, platform-independent, communitysupported software for describing and comparing microbial communities. Appl Environ Microbiol. 2009;75(23):7537-41. doi:10.1128/aem.01541-09. 